\title{
Analytic determination of high-order post-Newtonian self-force contributions to gravitational spin precession
}

\author{
Donato Bini ${ }^{1}$ and Thibault Damour ${ }^{2}$ \\ ${ }^{1}$ Istituto per le Applicazioni del Calcolo "M. Picone", CNR, I-00185 Rome, Italy \\ ${ }^{2}$ Institut des Hautes Etudes Scientifiques, 91440 Bures-sur-Yvette, France
}

(Dated: August 24, 2018)

\begin{abstract}
Continuing our analytic computation of the first-order self-force contribution to the "geodetic" spin precession frequency of a small spinning body orbiting a large (non-spinning) body we provide the exact expressions of the tenth and tenth-and-a-half post-Newtonian terms. We also introduce a new approach to the analytic computation of self-force regularization parameters based on a WKB analysis of the radial and angular equations satisfied by the metric perturbations.
\end{abstract}

\section{INTRODUCTION}

The impending prospect of detecting gravitationalwave signals from coalescing compact binary systems motivates renewed studies of the general relativistic dynamics of binary systems made of spinning bodies. It has been emphasized in Ref. [1] that a simple way of computing (to linear order in each spin) the spin-dependent interaction terms $H_{\text {int }}=\boldsymbol{\Omega}_{1}^{\mathrm{SO}} \cdot \mathbf{S}_{1}+\boldsymbol{\Omega}_{2}^{\mathrm{SO}} \cdot \mathbf{S}_{2}$ in the Hamiltonian of a binary system was to compute (when considering, say, the term linear in $\mathbf{S}_{1}$ ) the spin precession angular velocity of $\mathbf{S}_{1}$ in the gravitational field generated by the two masses $m_{1}, m_{2}$, and, eventually, the spin $\mathbf{S}_{2}$. Indeed, this spin precession angular velocity (which can be obtained by writing that $\mathbf{S}_{1}$ is parallely propagated along the world line of $m_{1}$ ) is simply equal to the coefficient $\mathbf{\Omega}_{1}^{\mathrm{SO}}$ of $\mathbf{S}_{1}$ in $H_{\mathrm{int}}$. On the other hand, it was recently remarked [2, 3] that, in the simple case of a binary moving on circular orbits, the (z-component of the) spin precession, $\Omega_{1}^{\mathrm{SO}}$, could be expressed in terms of the norm $|\nabla k|$ of the covariant derivative of the helical Killing vector $k=\partial_{t}+\Omega \partial_{\phi}$ characteristic of circular motions, namely

$$
\Omega_{1}^{\mathrm{SO}}=\Omega-|\nabla k|,
$$

where $\Omega$ denotes the orbital frequency. [The gaugeinvariant quantity $|\nabla k|$ can be viewed as a firstderivative-level generalization of Detweiler's redshift invariant [4], which is expressible in terms of the norm $|k|$ of the Killing vector $k$.]

The gauge-invariant functional relation between $\Omega_{1}^{\mathrm{SO}}$, or equivalently $|\nabla k|$, and the orbital frequency $\Omega$ has been recently studied (both numerically and analytically) in Refs. [2, 3]. In particular, we have derived (as part of a sequence of analytical gravitational self-force studies) in [3] the first-order self-force contribution (linear in the mass ratio $\left.q=m_{1} / m_{2} \ll 1\right)$ to the "geodetic" spin precession frequency $\Omega_{1}^{\mathrm{SO}}$ to the eight-and-a-half postNewtonian $(\mathrm{PN})$ order, i.e. up to terms of order $y^{8.5}$ included, where

$$
y=\left(\frac{G m_{2} \Omega}{c^{3}}\right)^{2 / 3}
$$

is a convenient dimensionless frequency parameter of order $O\left(1 / c^{2}\right)$. [We henceforth use, for simplicity, units where $G=c=1$.] As in [3] we restrict ourselves here to the case of a small spinning body $m_{1}, \mathbf{S}_{1}$, orbiting a large non-spinning body $m_{2}, \mathbf{S}_{2}=0$.

The aim of the present note is to report on an extension of our previous analytical computation of spin precession to the $10.5 \mathrm{PN}$ level, i.e. up to terms of order $y^{10.5}$ included. This extension was motivated by private communications from Dolan et al. [5] who pointed out apparent discrepancies (starting at level $O\left(y^{7}\right)$ ) between some of their high-accuracy numerical results (see Table III in Ref. [6]) and our published 8.5PN analytical results. These discrepancies led us to carefully re-examine our previous computations, and to push them to higher PN orders. We so discovered that, though all our basic analytical building blocks were correct, their manipulation by an algebraic software led to some instabilities (due to the length of the analytical expressions at high PN orders), that had led to a few errors in our final results. More precisely, the rational term, among the seven (transcendental) contributions to the coefficient of $y^{7}$, was incorrectly obtained, and, in the coefficient of $y^{8}$ (which contains fifteen different contributions), both the rational term and the coefficient of $\pi^{2}$ were incorrectly obtained. Correspondingly, there were errors in the (rational) coefficients of $y^{7}$ and $y^{8}$ in the subtraction term $B(y)$. [See detailed results below.] After having found these errors, corrected them, and communicated the corrections to Dolan et al., the latter authors confirmed that our $O\left(y^{8}\right)$ corrected results were now in satisfactory agreement with their high-accuracy numerical results. [More recently, Shah 7] independently pointed out to us the three discrepant coefficients mentioned above, which we had already analytically derived, and which he and his collaborators had independently derived by using the numerical-analytical method of Ref. [8].]

\section{TECHNICAL REMINDERS}

Let us recall the notation and main technical results of Ref. [3] that we shall need to express our new results. We consider a two-body system of masses $m_{1}$ and $m_{2}$, moving along circular orbits, in the limit $m_{1} \ll m_{2}$. Here we only endow the small mass $m_{1}$ with spin $S_{1}$, keeping the 
large mass $m_{2}$ non-spinning. This means that one is dealing with linear perturbations $h_{\mu \nu}\left(x^{\lambda}\right)$ of a Schwarzschild background of mass $m_{2}$ by a small mass $m_{1}$, moving on a circular orbit of radius $r_{0}$. As emphasized by Detweiler [4], the perturbed metric admits the helical Killing vector $k=\partial_{t}+\Omega \partial_{\phi}$, i.e., the metric perturbation depends only on $\bar{\phi}=\phi-\Omega t, r$ and $\theta, h_{\mu \nu}(\bar{\phi}, r, \theta)$.

The four-velocity of $m_{1}$, normalized with respect to the metric $g_{\mu \nu}^{R}\left(x^{\lambda}\right)=g_{\mu \nu}^{(0)}+q h_{\mu \nu}^{\mathrm{R}}+O\left(q^{2}\right)$, (here $q \equiv$ $m_{1} / m_{2} \ll 1$ and the superscript $R$ indicates the regular part [9] of $h_{\mu \nu}\left(x^{\lambda}\right)$ around the world line of $\left.m_{1}\right)$, can be written as

$$
U_{1}^{\mu}=\frac{k^{\mu}}{|k|} \equiv \Gamma k^{\mu}, \quad \Gamma \equiv \frac{1}{|k|},
$$

where (to linear order in $q$ )

$$
\begin{aligned}
|k| & =\sqrt{\left[-g_{\mu \nu}^{\mathrm{R}} k^{\mu} k^{\nu}\right]_{1}}=\sqrt{1-\frac{2 m_{2}}{r_{0}}-\Omega^{2} r_{0}^{2}-q h_{k k}} \\
& =\sqrt{1-\frac{2 m_{2}}{r_{0}}-\Omega^{2} r_{0}^{2}}\left(1-\frac{1}{2} q \frac{h_{k k}}{1-\frac{2 m_{2}}{r_{0}}-\Omega^{2} r_{0}^{2}}\right)
\end{aligned}
$$

with $h_{k k}=\left[h_{\mu \nu}^{\mathrm{R}}(x) k^{\mu} k^{\nu}\right]_{1}$. Writing that $m_{1}$ moves along an equatorial circular geodesic yields the conditions $\partial_{\mu} g_{k k}^{R}=0$, which lead to [4]

$$
\begin{aligned}
\Omega & =\sqrt{\frac{m_{2}}{r_{0}^{3}}}\left(1-q \frac{r_{0}^{2}}{4 m_{2}}\left[\partial_{r} h_{k k}^{\mathrm{R}}\right]_{1}\right), \\
{\left[\partial_{\bar{\phi}} h_{k k}^{\mathrm{R}}\right]_{1} } & =0 .
\end{aligned}
$$

Eq. (2.3) allows one to trade the gauge-dependent radius $r_{0}$ for the gauge-invariant dimensionless frequency parameter $y$, Eq. (1.2), using

$$
\begin{aligned}
r_{0} & =\frac{m_{2}}{y}-q \frac{m_{2}^{2}}{6 y^{3}}\left[\partial_{r} h_{k k}^{\mathrm{R}}\right]_{1}, \\
\frac{m_{2}}{r_{0}} & =y\left(1+q \frac{m_{2}}{6 y^{2}}\left[\partial_{r} h_{k k}^{\mathrm{R}}\right]_{1}\right) .
\end{aligned}
$$

The geodetic spin-orbit precession frequency along the world line of $m_{1}$ has, as only nonvanishing component, $\Omega_{1}^{\mathrm{SO}} \equiv \Omega_{z}^{\mathrm{SO}}$ given by Eq. (1.1) above. In this equation, the norm $|\nabla k|$ of the covariant derivative of the helical Killing vector $k=\partial_{t}+\Omega \partial_{\phi}$ is defined as

$$
|\nabla k|^{2}=\frac{1}{2}\left(\nabla_{\mu} k_{\nu}\right)\left(\nabla^{\mu} k^{\nu}\right)
$$

where all tensorial operations are done with the metric $g_{\mu \nu}^{\mathrm{R}}(x)$. The explicit expression of $|\nabla k|$ can be written as

$$
|\nabla k|=|\nabla k|^{(0)}\left(1+q \delta(y)+O\left(q^{2}\right)\right),
$$

where

$$
|\nabla k|^{(0)}=\Omega \sqrt{1-3 y}
$$

is the well-known result for gyroscopic precession (with respect to a rotating, polar-coordinate frame) in a Schwarzschild background [10], and where

$$
\begin{aligned}
\delta(y)= & -\frac{1}{2}(1-2 y) h_{r r}-\frac{y^{2}(1-y)}{2 m_{2}^{2}(1-2 y)} h_{\phi \phi} \\
& -\frac{y^{3 / 2}}{m_{2}(1-2 y)} h_{t \phi}-\frac{y}{2(1-2 y)(1-3 y)} h_{k k} \\
& -\frac{1}{2 \sqrt{y}}\left(\partial_{\phi} h_{r k}-\partial_{r} h_{\phi k}\right) .
\end{aligned}
$$

In Eq. (2.9) all quantities are to be regularized and evaluated for $\theta=\pi / 2$.

The quantity $\delta(y)$, which measures the fractional first order self-force (1SF) correction to $|\nabla k|$, is equivalent to the quantity $\delta \psi(y)$ which measures the $1 \mathrm{SF}$ contribution to the dimensionless ratio [2]

$\psi(y) \equiv \frac{\Omega_{1}^{\mathrm{SO}}}{\Omega}=1-\frac{|\nabla k|}{\Omega}=1-\sqrt{1-3 y}\left[1+q \delta(y)+O\left(q^{2}\right)\right]$

Explicitly, we have

$$
\delta \psi(y)=-\sqrt{1-3 y} \delta(y)
$$

Following the methodology explained in Refs. [11 15], and extending the results of Ref. [3] to higher postNewtonian orders (by using radiative solutions, $X_{(\text {in) }}$, $X_{(\text {up })}$, up to $\left.l=7\right)$, we have computed $\delta(y)$ up to or$\operatorname{der} y^{10.5}$.

\section{NEW HIGHER POST-NEWTONIAN TERMS IN $\delta(y)$ AND $\delta \psi(y)$}

Before listing the complete expressions of $\delta(y)$ and $\delta \psi(y)$ to order $y^{10.5}$ let us indicate that our previous $O\left(y^{8.5}\right)$-accurate results missed one term at level $y^{7}$ and two terms at level $y^{8}$, while the $y^{7.5}$ and $y^{8.5}$ terms were complete.

More precisely, the correct $O\left(y^{8.5}\right)$-accurate expression of $\delta(y)$ is obtained by adding $\Delta c_{7}^{\delta} y^{7}+\Delta c_{8}^{\delta} y^{8}$ to Eq. (4.33) in [3], where

$$
\begin{aligned}
\Delta c_{7}^{\delta} & =-\frac{1485630311863}{45831035250}, \\
\Delta c_{8}^{\delta} & =\frac{8}{8505} \pi^{2}-\frac{25377697082469367}{262980313505910} .
\end{aligned}
$$

Equivalently, the correct $O\left(y^{8.5}\right)$-accurate expression of $\delta \psi(y)$ is obtained by adding $\Delta c_{7}^{\delta \psi} y^{7}+\Delta c_{8}^{\delta \psi} y^{8}$ to Eq. (5.4) in [3], where $\Delta c_{7}^{\delta \psi}=-\Delta c_{7}^{\delta}$ and $\Delta c_{8}^{\delta \psi}=-\Delta c_{8}^{\delta}+\frac{3}{2} \Delta c_{7}^{\delta}$, i.e.,

$$
\begin{aligned}
\Delta c_{7}^{\delta \psi} & =\frac{1485630311863}{45831035250} \\
\Delta c_{8}^{\delta \psi} & =-\frac{8}{8505} \pi^{2}+\frac{629539392522290711}{13149015675295500}
\end{aligned}
$$


The full $O\left(y^{10.5}\right)$-accurate expressions of $\delta(y)$ and $\delta \psi(y)$ read

$$
\begin{aligned}
& \delta(y)=-y^{2}+\frac{3}{2} y^{3}+\frac{69}{8} y^{4}+\left(\frac{53321}{240}+\frac{496}{15} \ln (2)+16 \gamma+8 \ln (y)-\frac{20471}{1024} \pi^{2}\right) y^{5} \\
& +\left(\frac{15462423}{4480}+\frac{172}{5} \gamma+\frac{1436}{105} \ln (2)-\frac{357521}{1024} \pi^{2}+\frac{86}{5} \ln (y)+\frac{729}{14} \ln (3)\right) y^{6} \\
& +\frac{26536}{1575} y^{13 / 2} \pi \\
& +\left(\frac{16156122817}{1209600}-\frac{30832}{105} \gamma-\frac{3344}{21} \ln (2)-\frac{512537515}{393216} \pi^{2}-\frac{15416}{105} \ln (y)-\frac{40581}{140} \ln (3)+\frac{1407987}{524288} \pi^{4}\right) y^{7} \\
& +\frac{670667}{22050} y^{15 / 2} \pi \\
& +\left(-\frac{41432062371919}{2540160000}+\frac{96697099}{141750} \gamma-\frac{58208}{105} \ln (2)^{2}-\frac{1291394011}{3638250} \ln (2)-\frac{1007542476707}{353894400} \pi^{2}\right. \\
& +\frac{9765625}{28512} \ln (5)+\frac{96697099}{283500} \ln (y)+\frac{2364633}{12320} \ln (3)-\frac{856}{25} \ln (y)^{2}+\frac{162286431837}{335544320} \pi^{4}-\frac{869696}{1575} \ln (2) \gamma \\
& \left.+\frac{1344}{5} \zeta(3)-\frac{3424}{25} \gamma \ln (y)-\frac{3424}{25} \gamma^{2}-\frac{434848}{1575} \ln (2) \ln (y)\right) y^{8} \\
& -\frac{3872542979}{13097700} y^{17 / 2} \pi \\
& +\left(-\frac{4084955265168837911}{1173553920000}+\frac{118580138377}{14553000} \gamma+\frac{45728}{1225} \ln (2)^{2}+\frac{58794404629417}{3972969000} \ln (2)\right. \\
& +\frac{7776}{5} \zeta(3)-\frac{100335874551071}{26424115200} \pi^{2}-\frac{20486328125}{5189184} \ln (5)+\frac{118580138377}{29106000} \ln (y) \\
& -\frac{32288}{75} \gamma^{2}+\frac{143985009429}{15695680} \ln (3)-8072 / 75 \ln (y)^{2}+\frac{773697968441461}{21474836480} \pi^{4} \\
& -\frac{28431}{49} \ln (y) \ln (3)-\frac{56862}{49} \gamma \ln (3)-\frac{56862}{49} \ln (2) \ln (3) \\
& \left.-\frac{1210816}{2205} \ln (2) \gamma-\frac{32288}{75} \gamma \ln (y)-\frac{605408}{2205} \ln (2) \ln (y)-\frac{28431}{49} \ln (3)^{2}\right) y^{9} \\
& +\left(\frac{460314955849127}{524431908000} \pi-\frac{6228256}{11025} \ln (2) \pi-\frac{23264368}{165375} \pi \ln (y)+\frac{434848}{4725} \pi^{3}-\frac{46528736}{165375} \pi \gamma\right) y^{19 / 2} \\
& +\left(-\frac{5405869945189728461825461}{169160756244480000}-\frac{164976460027543}{15891876000} \gamma-\frac{4653978748}{467775} \ln (2)^{2}\right. \\
& -\frac{164366211989143}{31783752000} \ln (y)+\frac{144656188561370737}{4719887172000} \ln (2)+\frac{1337603}{2205} \ln (y)^{2}-\frac{489993464291995}{532710162432} \pi^{2} \\
& -\frac{80728}{21} \zeta(3)+\frac{5350412}{2205} \gamma \ln (y)-\frac{1169541476}{3274425} \ln (2) \ln (y)-\frac{2339082952}{3274425} \ln (2) \gamma+\frac{5350412}{2205} \gamma^{2} \\
& +\frac{86209353}{26950} \ln (3)^{2}-\frac{167271372501741}{3453049600} \ln (3)+\frac{161107421875}{10378368} \ln (5)+\frac{906012273831305533}{2748779069440} \pi^{4} \\
& \left.-\frac{21138410295}{134217728} \pi^{6}+\frac{86209353}{26950} \ln (y) \ln (3)+\frac{86209353}{13475} \gamma \ln (3)+\frac{86209353}{13475} \ln (2) \ln (3)+\frac{678223072849}{370656000} \ln (7)\right) y^{10} \\
& +\left(\frac{1242850565271443431}{159077678760000}+\frac{3164198}{6615} \pi^{2}-\frac{60364562}{77175} \gamma-\frac{26484566}{55125} \ln (2)-\frac{369603}{343} \ln (3)-\frac{30182281}{77175} \ln (y)\right) \pi y^{21 / 2} \\
& +O_{\ln }\left(y^{11}\right) \text {. }
\end{aligned}
$$




$$
\begin{aligned}
& \delta \psi=y^{2}-3 y^{3}-\frac{15}{2} y^{4}+\left(-\frac{6277}{30}-\frac{496}{15} \ln (2)-16 \gamma-8 \ln (y)+\frac{20471}{1024} \pi^{2}\right) y^{5} \\
& +\left(-\frac{87055}{28}+\frac{3772}{105} \ln (2)-\frac{52}{5} \gamma-\frac{26}{5} \ln (y)+\frac{653629}{2048} \pi^{2}-\frac{729}{14} \ln (3)\right) y^{6} \\
& -\frac{26536}{1575} y^{13 / 2} \pi \\
& +\left(-\frac{149628163}{18900}+\frac{7628}{21} \gamma+\frac{3814}{21} \ln (y)+\frac{4556}{21} \ln (2)+\frac{12879}{35} \ln (3)-\frac{1407987}{524288} \pi^{4}+\frac{297761947}{393216} \pi^{2}\right) y^{7} \\
& -\frac{113411}{22050} y^{15 / 2} \pi \\
& +\left(-\frac{74909462}{70875} \gamma+\frac{58208}{105} \ln (2)^{2}+\frac{340681718}{1819125} \ln (2)+\frac{164673979457}{353894400} \pi^{2}\right. \\
& -\frac{160934764317}{335544320} \pi^{4}-\frac{1344}{5} \zeta(3)+\frac{869696}{1575} \ln (2) \gamma+\frac{3424}{25} \gamma^{2}-\frac{199989}{352} \ln (3) \\
& -\frac{9765625}{28512} \ln (5)+\frac{856}{25} \ln (y)^{2}+\frac{434848}{1575} \ln (2) \ln (y)-\frac{37454731}{70875} \ln (y) \\
& \left.+\frac{403109158099}{9922500}+\frac{3424}{25} \gamma \ln (y)\right) y^{8} \\
& +\frac{1179591206}{3274425} y^{17 / 2} \pi \\
& +\left(-\frac{4454779894}{606375} \gamma-\frac{1064368}{1225} \ln (2)^{2}-\frac{138895624334}{9029475} \ln (2)-\frac{22832200546571}{8808038400} \pi^{2}\right. \\
& -\frac{758053590944149}{21474836480} \pi^{4}-1152 \zeta(3)-\frac{3077728}{11025} \ln (2) \gamma+\frac{3376}{15} \gamma^{2}+\frac{28431}{49} \ln (3)^{2} \\
& -\frac{71602663581}{7847840} \ln (3)+\frac{11576171875}{2594592} \ln (5)+\frac{844}{15} \ln (y)^{2}+\frac{28431}{49} \ln (y) \ln (3) \\
& -\frac{1538864}{11025} \ln (2) \ln (y)-\frac{2227389947}{606375} \ln (y)+\frac{56862}{49} \gamma \ln (3)+\frac{3985926908910281}{1146048750} \\
& \left.+\frac{56862}{49} \ln (2) \ln (3)+\frac{3376}{15} \gamma \ln (y)\right) y^{9} \\
& +\left(-\frac{660044682996077}{524431908000} \pi-\frac{434848}{4725} \pi^{3}+\frac{46528736}{165375} \pi \gamma+\frac{6228256}{11025} \ln (2) \pi\right. \\
& \left.+\frac{23264368}{165375} \pi \ln (y)\right) y^{19 / 2} \\
& +\left(\frac{11467229058074}{496621125} \gamma+\frac{21138410295}{134217728} \pi^{6}+\frac{30719079112}{3274425} \ln (2)^{2}-\frac{1306135539288758}{147496474125} \ln (2)\right. \\
& -\frac{152033994681460553}{13317754060800} \pi^{2}-\frac{755954175166870909}{2748779069440} \pi^{4}+\frac{680336}{105} \zeta(3)-\frac{478423984}{654885} \ln (2) \gamma \\
& -\frac{35570296}{11025} \gamma^{2}-\frac{54832464}{13475} \ln (3)^{2}+\frac{214411899501351}{3453049600} \ln (3)-\frac{437134765625}{20756736} \ln (5) \\
& -\frac{8892574}{11025} \ln (y)^{2}-\frac{54832464}{13475} \ln (y) \ln (3)-\frac{239211992}{654885} \ln (2) \ln (y)+\frac{5724079403437}{496621125} \ln (y) \\
& -\frac{109664928}{13475} \gamma \ln (3)+\frac{552424223705497767347}{20649506377500}-\frac{109664928}{13475} \ln (2) \ln (3)-\frac{35570296}{11025} \gamma \ln (y) \\
& \left.-\frac{678223072849}{370656000} \ln (7)\right) y^{10} \\
& +\left(-\frac{178279193702345741}{26512946460000} \pi-\frac{11255086}{33075} \pi^{3}+\frac{46324078}{128625} \pi \gamma-\frac{2889622}{7875} \ln (2) \pi\right. \\
& \left.+\frac{369603}{343} \pi \ln (3)+\frac{23162039}{128625} \pi \ln (y)\right) y^{21 / 2}+O_{\ln }\left(y^{11}\right) \text {. }
\end{aligned}
$$


Note that, in the above expressions, we used the computer-algebra-related notation $\ln (a)^{n}$ to denote $\ln ^{n}(a)$ and $O_{\ln }\left(y^{11}\right)$ to denote a term of order $y^{11} \ln ^{n} y$ for some $n$. The corresponding $O_{\ln }\left(u^{11}\right)$-accurate expansion of the effective gyrogravitomagnetic ration $g_{S_{*}}^{1 \mathrm{SF}}(u)$ is given in the Appendix.

\section{ANALYTIC EXPRESSION OF THE SUBTRACTION TERM}

Prompted by Dolan et al. [5], who pointed out discrepancies at order $y^{7}$ and $y^{8}$ between our Eq. (4.30) in [3] and their (unpublished) corresponding expression for the subtraction term $B(y)$, we have found a way to derive an exact analytic expression for $B(y)$ within our formalism, which is based on Regge-Wheeler-Zerilli-type tensorial multipolar expansions. As we shall now explain, our derivation is a novel approach grounded on a Wentzel-Kramers-Brillouin (WKB) analysis of the homogeneous radial (Regge-Wheeler, RW) equation satisfied by the fundamental building blocks, $X_{\text {in }}$ and $X_{\mathrm{up}}$, of our formalism. This WKB approach (which we explain in detail below) is quite different from the approach traditionally used in gravitational self-force theory, which is based on local, Hadamard-type expansions of the metric $h_{\mu \nu}$, in Lorenz-gauge, near the world line of $m_{1}$ (see e.g., $[9,[16,17])$. In addition, our approach defines the subtraction terms by considering the limit $l \rightarrow \infty$ where $l$ denotes the degree in a tensorial multipolar expansion, while the usual self-force calculations define subtraction terms by considering a limit $l_{s} \rightarrow \infty$, where $l_{s}$ denotes the order in a scalar multipolar expansion. One can show that, for the quantities we shall consider, the two different limiting procedures should give the same subtraction terms at leading order. [However, at higher orders in local singularity expansions, the extension ambiguities of such expansions do not imply anymore their equivalence.]

Let us start by recalling the form of the WKB approximation of the solutions of a one-dimensional Schrödinger equation, say

$$
\frac{d^{2}}{d x^{2}} \Psi=\frac{Q(x)}{\hbar^{2}} \Psi(x) .
$$

The WKB solutions of Eq. (4.1) are written in the form

$$
\Psi(x)=e^{\frac{S_{0}}{\hbar}+S_{1}+O(\hbar)} .
$$

As indicated here, it will be sufficient for our purpose to keep only the leading and next-to-leading terms in the WKB expansion. At this order of approximation, the two independent solutions of Eq. (4.1) read

$$
\Psi_{ \pm}(x)=C_{ \pm} \frac{e^{ \pm \int p(x) d x}}{\sqrt{p(x)}}, \quad p(x)=\sqrt{Q(x)},
$$

corresponding to

$$
\frac{S_{0}}{\hbar}= \pm \int p(x) d x, \quad S_{1}=-\frac{1}{2} \ln p(x) .
$$

The choice $C_{ \pm}=1 / \sqrt{2}$ would imply that the Wronskian of these solutions is 1 :

$$
W=\Psi_{-} \Psi_{+}^{\prime}-\Psi_{+} \Psi_{-}^{\prime}=1 .
$$

Note that we will use the WKB approximation in the classically forbidden domain, where $Q(x)$ is positive so that the solutions $\Psi_{ \pm}$are exponentially growing or decaying.

We first apply this approximation to the (homogeneous) radial RW equation

$$
\frac{d^{2}}{d r_{*}^{2}} X=\left[f(r)\left(\frac{l(l+1)}{r^{2}}-\frac{6 M \eta^{2}}{r^{3}}\right)-\eta^{2} m^{2} \Omega^{2}\right] X .
$$

Here, $\eta \equiv 1 / c, m$ is the spherical harmonics order, $\Omega$ denotes the orbital frequency, and

$$
d r_{*}=\frac{d r}{f(r)}, \quad f(r)=1-\frac{2 M \eta^{2}}{r} .
$$

The spatial variable (denoted $x$ in Eq.44.1) in this onedimensional Schrödinger equation is $r_{*}$, while we shall take as small expansion parameter $\hbar$ the quantity

$$
\hbar \equiv \frac{1}{L},
$$

where we introduced the convenient notation

$$
L=l+\frac{1}{2} .
$$

Note indeed that the coefficient $l(l+1)$ in the centrifugal potential can be written as

$$
l(l+1)=L^{2}-\frac{1}{4},
$$

and is of order $\sim \frac{1}{\hbar^{2}}$.

In order to capture the near-world-line singularity expansion within our tensorial multipolar expansion, we need to consider a limit where both $l \sim L$ and $m$ tend to infinity with the ratio $w \equiv m / L$ being kept fixed. In this limit the two dominant terms (of order $1 / \hbar^{2}$ ) in Eq. (4.1) are

$$
\begin{aligned}
\frac{Q_{*}}{\hbar^{2}} & =l(l+1)\left[\frac{1}{r^{2}} f(r)-\eta^{2} \frac{m^{2}}{l(l+1)} \Omega^{2}\right]+O\left(L^{0}\right) \\
& =L^{2}\left[\frac{1}{r^{2}} f(r)-\eta^{2} \frac{m^{2}}{L^{2}} \Omega^{2}\right]+O\left(L^{0}\right) \\
& =L^{2}\left[\frac{1}{r^{2}} f(r)-\eta^{2} w^{2} \Omega^{2}\right]+O\left(L^{0}\right)
\end{aligned}
$$

Correspondingly to the accuracy used in Eq. (4.2), we can neglect the terms of order $O\left(L^{0}\right)$ in the above equation, which notably means neglecting the term $6 \mathrm{M} / \mathrm{r}^{3}$ in Eq. (4.6). At this stage no expansion is performed in the PN-parameter $\eta=1 / c$.

Introducing the notation

$$
\Delta(r)=1-\frac{2 M \eta^{2}}{r}-\eta^{2} w^{2} \Omega^{2} r^{2}
$$


we have

$$
Q_{*}=p_{*}^{2}=L^{2} \frac{\Delta(r)}{r^{2}}, \quad p_{*}=L \frac{\sqrt{\Delta(r)}}{r},
$$

so that the building blocks of the WKB solution (4.3) read

$$
\frac{S_{0}}{\hbar}= \pm \int p_{*} d r_{*}= \pm \int p_{*} \frac{d r}{f}= \pm L \int \frac{\sqrt{\Delta(r)}}{f(r)} \frac{d r}{r},
$$

and

$$
S_{1}=-\frac{1}{2} \ln p_{*} .
$$

More explicitly

$$
\sqrt{p_{*}}=\sqrt{L} \frac{\Delta^{1 / 4}}{\sqrt{r}}
$$

so that

$$
\frac{C_{ \pm}}{\sqrt{p_{*}}}=\tilde{C}_{ \pm} \frac{\sqrt{r}}{\Delta^{1 / 4}}
$$

where we have re-absorbed the factor $\sqrt{L}$ in the constant $C_{ \pm}\left[\tilde{C}_{ \pm}=C_{ \pm} / \sqrt{L}\right]$.

The final result of this WKB analysis is that two independent solutions of the RW equation (4.6) are

$$
X_{ \pm}=\tilde{C}_{ \pm} \frac{\sqrt{r}}{\Delta^{1 / 4}} e^{ \pm L \int \frac{\sqrt{\Delta}}{f} \frac{d r}{r}} .
$$

We checked that the PN expanded solutions of the RW equation that we constructed in our formalism [11 15] agree with those WKB solutions, with the following correspondence

$$
\begin{aligned}
X_{(\mathrm{in})} & \approx \frac{\sqrt{r}}{\Delta^{1 / 4}} e^{L \int \frac{\sqrt{\Delta}}{r f} d r}, \\
X_{(\text {up })} & \approx \frac{\sqrt{r}}{\Delta^{1 / 4}} e^{-L \int \frac{\sqrt{\Delta}}{r f} d r} .
\end{aligned}
$$

Note for instance that, when expanding in powers of $\eta$ the right-hand-side (rhs) of $X_{(\mathrm{in})}$, as given in Eq. (4.19), its leading order is $\sqrt{r} e^{L \ln r}=r^{l+1}$ in agreement with the normalization of our PN solution which was chosen as

$$
X_{(\mathrm{in})}^{l \omega}(r)=r^{l+1}\left(1+A^{l \omega}(r)\right)
$$

with $A^{l \omega}(r)=O\left(\eta^{2}\right)$.

Inserting the above WKB solutions for $X_{(\text {in) }}$ and $X_{(\text {up })}$ in the analytical expressions for $\delta_{l m}^{ \pm(\text {odd/even) }}$ given in Ref. [3] [see Eqs. (4.10) and (4.12) together with Eqs. (4.11), (4.13) and (4.23) there] yields expressions for $\delta_{l}^{ \pm}(y)=$ $\delta_{l}^{ \pm, \text {even }}(y)+\delta_{l}^{ \pm \text {odd }}(y)$ of the form of Eq. (4.28) there, i.e.,

$$
\delta_{l}^{ \pm}(y) \equiv \sum_{m} \delta_{l m}^{ \pm}(y)= \pm L A(y)+B(y)+O\left(\frac{1}{L^{2}}\right) .
$$

At this stage the subtraction term $B(y)$ is given by a sum over $m$ of the form

$$
\sum_{m} f\left(\frac{m}{L}\right)\left|Y_{l m}\right|^{2}+g\left(\frac{m}{L}\right)\left|\frac{d Y_{l m}}{d \theta}\right|^{2},
$$

where $Y_{l m}(\theta, \phi)$ and its $\theta$-derivative are both evaluated at $\theta=\pi / 2$ (and $\phi=0)$. Such a sum can be asymptotically evaluated, in the limit $L \rightarrow \infty$ with $m / L$ fixed, in terms of an integral, between -1 and 1 , over the variable $w=m / L$. In order to do so one needs asymptotic estimates for $\left|Y_{l m}\right|^{2}$ and $\left|\frac{d Y_{l m}}{d \theta}\right|^{2}$ as functions of $w$ in the large $L$ limit. Such asymptotic estimates can be derived by a WKB analysis of the $\theta$ differential equation satisfied by $\Theta_{l m}(\theta)$ (defined by factoring $\left.Y_{l m}(\theta, \phi)=\Theta_{l m}(\theta) e^{i m \phi}\right)$. Indeed, $\Theta_{l m}(\theta)$ satisfies a onedimensional Schrödinger equation of the type (4.1), when using the variable $\lambda=\int_{\pi / 2} d \theta / \sin \theta$, namely

$$
\frac{d^{2}}{d \lambda^{2}} \Theta_{l m}=-P^{2}(\lambda) \Theta_{l m},
$$

with

$$
P^{2}(\lambda)=l(l+1) \sin ^{2} \theta(\lambda)-m^{2} .
$$

This leads to WKB solutions of the type

$$
\Theta_{l m}(\lambda)=C_{+} \frac{e^{i \int P(\lambda) d \lambda}}{\sqrt{P(\lambda)}}+C_{-} \frac{e^{-i \int P(\lambda) d \lambda}}{\sqrt{P(\lambda)}},
$$

for appropriate choices of the constants $C_{ \pm}$determined by regularity conditions at $\lambda=-\infty$ (corresponding to $\theta=0)$ and $\lambda=+\infty$ (corresponding to $\theta=\pi$ ). When evaluating $\Theta_{l m}(\lambda)$ and $\frac{d}{d \lambda} \Theta_{l m}(\lambda)=\sin \theta \frac{d}{d \theta} \Theta_{l m}$ at $\theta=$ $\pi / 2$ (i.e., $\lambda=0$ ) one finds the following WKB estimates

$$
\left|Y_{l m}\left(\frac{\pi}{2}, 0\right)\right|^{2} \approx \frac{1}{\pi^{2}} \frac{1}{\sqrt{1-w^{2}}} \delta_{l-m}^{\text {even }},
$$

and

$$
\left|\partial_{\theta} Y_{l m}\left(\frac{\pi}{2}, 0\right)\right|^{2} \approx \frac{L^{2}}{\pi^{2}} \sqrt{1-w^{2}} \delta_{l-m}^{\text {odd }} .
$$

Here $\delta_{l-m}^{\text {even }}\left(\delta_{l-m}^{\text {odd }}\right)$ is equal to 1 when $l-m$ is even (odd) and to 0 otherwise. The above estimates are not a priori uniformly valid in the full range $-l \leq m \leq l$ because our WKB analysis requires $L^{2}-m^{2} \gg 1$. However, they can correctly evaluate the asymptotic values of the integrals that we shall be interested in below (which have only a relatively small contribution from the neighborhoods of the boundary points $w= \pm 1$ ). We have checked the estimates (4.26) and (4.27) by using the explicit expressions of $Y_{l m}\left(\frac{\pi}{2}, 0\right)$ and $\partial_{\theta} Y_{l m}\left(\frac{\pi}{2}, 0\right)$ given in Eqs. (32) and (33) of [18] . A consequence of Eqs. (4.26) and (4.27) is that

$$
\begin{gathered}
\sum_{m} \frac{4 \pi}{2 l+1}\left|Y_{l m}\left(\frac{\pi}{2}, 0\right)\right|^{2} f\left(\frac{m}{L}\right) \approx \frac{1}{\pi} \int_{-1}^{1} \frac{d w}{\sqrt{1-w^{2}}} f(w), \\
\sum_{m} \frac{4 \pi}{2 l+1} \frac{\left|\partial_{\theta} Y_{l m}\left(\frac{\pi}{2}, 0\right)\right|^{2}}{L^{2}} g\left(\frac{m}{L}\right) \approx \frac{1}{\pi} \int_{-1}^{1} d w \sqrt{1-w^{2}} g(w)
\end{gathered}
$$


As an example of the application of these asymptotic estimates we have computed the analytic expression of the $L \rightarrow \infty$ limit of the first-order self-force redshift quantity $h_{k k}$. Starting from Eqs. (29) and (30) of Ref. [11] one finds that $B_{h_{k k}} \equiv \lim _{l \rightarrow \infty}\left(h_{k k, l m}^{(\text {even })}+h_{k k, l m}^{(\text {odd })}\right)$ is given by

$$
\begin{aligned}
B_{h_{k k}} & =\frac{2 y(1-3 y)^{3 / 2}}{\sqrt{1-2 y}} \frac{1}{\pi} \int_{-1}^{1} \frac{d w}{\sqrt{\left(1-w^{2}\right)\left(1-k^{2} w^{2}\right)}} \\
& =\frac{2 y(1-3 y)^{3 / 2}}{\sqrt{1-2 y}} \frac{2}{\pi} \operatorname{EllipticK}(k),
\end{aligned}
$$

where

$$
k^{2}=\frac{y}{1-2 y}
$$

and where EllipticK $(k)$ denotes the complete elliptic in- tegral of the first kind (with $w \equiv \sin \alpha$ ):

$$
\operatorname{EllipticK}(k)=\int_{0}^{\pi / 2} \frac{d \alpha}{\sqrt{1-k^{2} \sin ^{2} \alpha}} .
$$

This result agrees with the subtraction term obtained by the usual self-force Hadamard-type analysis [4, 17], i.e., the term denoted $\tilde{D}_{0}=(1-3 y) D_{0}$ in $[11,12$. [Note that there is a misprint in the last term of Eq. (56) in [12]; the coefficient of $u^{7}$ should read $\left.+4409649 / 524288\right]$.

When applying the above WKB asymptotic estimates (for both the radial functions $X_{ \pm}(r)$ and the angular functions $Y_{l m}$ and $\left.\partial_{\theta} Y_{l m}\right)$ to the $l \rightarrow \infty$ limit of the quantity $\delta_{l}^{ \pm}(y)$, (4.21), we obtain the following analytic expression for the $O\left(L^{0}\right)$ subtraction term $B(y)$

$$
B_{\mathrm{WKB}}(y)=\frac{1}{\pi} \sqrt{\frac{1-3 y}{1-2 y}}[(4-9 y) \operatorname{EllipticE}(k)-2(2-5 y) \operatorname{EllipticK}(k)] .
$$

Here EllipticE $(k)$ denotes the complete elliptic integral of the second kind

$$
\operatorname{EllipticE}(k)=\int_{0}^{\pi / 2} d \alpha \sqrt{1-k^{2} \sin ^{2} \alpha} .
$$

The expansion in powers of $y$ of $B_{\mathrm{WKB}}$ reads, up to the $11 \mathrm{PN}$ level

$$
\begin{aligned}
B_{\mathrm{WKB}}(y)= & -\frac{1}{2} y+\frac{1}{4} y^{2}+\frac{63}{128} y^{3}+\frac{995}{1024} y^{4}+\frac{63223}{32768} y^{5} \\
& +\frac{126849}{32768} y^{6}+\frac{16567767}{2097152} y^{7}+\frac{555080733}{33554432} y^{8} \\
& +\frac{77104836855}{2147483648} y^{9}+\frac{350273500199}{4294967296} y^{10} \\
& +\frac{26812467118879}{137438953472} y^{11}+O\left(y^{12}\right) .
\end{aligned}
$$

In our previous work [3] the subtraction term $B$ was not derived independently of our computation of $\delta_{l m}^{ \pm}$but was obtained from the large $l$ limit of the PN expanded version of $\delta_{l m}^{ \pm}$. The algebraic-manipulation errors mentioned above induced corresponding errors in our previous evaluation of the PN expansion of $B$ (as pointed out to us by Dolan et al. [5]). More explicitly, the coefficients of $y^{7}$ and $y^{8}$ in Eq. (4.30) in [3] were in error, and Eq. (4.34) gives instead their correct values.

\section{CONCLUDING REMARKS}

The analytic computation of the post-Newtonian expansion of the first-order self-force contribution to spin precession has been raised here to the ten and ten-andhalf post-Newtonian level.
Our analysis has also corrected two terms (at the PN levels 7 and 8) among our previous 8.5 PN-accurate calculation of spin-orbit effects [3]. More precisely, we have shown that Eq. (4.33) in [3] needs to be augmented by the two terms in Eq. (3.1). Equivalently, Eq. (5.4) in [3] needs to be augmented by the two terms in Eq. (3.2). These missing terms were caused by algebraic errors in the manipulation of large analytic expressions. Note that these errors affected only a few terms among many contributions (essentially only rational terms). The missing contributions to the coefficients of $y^{7}$ and $y^{8}$ in $\delta \psi(y)$ are numerically equal to $\Delta c_{7}^{\psi}=32.41537757$ and $\Delta c_{8}^{\psi}=47.86801827$. These values are rather small compared to the corresponding typical values of the general PN coefficient $c_{n}^{\psi} \sim-0.12 \times 3^{n}$ (linked to the pole singularity of $\delta \psi(y)$ at $y=\frac{1}{3}$, see Eq. (5.14) in [3]). The fractional modifications brought to the coefficients $g_{6}^{c}$ and $g_{7}^{c}$ in Eqs. (6.36), (6.37) and (6.38) are correspondingly small, $\delta g_{6} / g_{6} \simeq-0.02487821950$ and $\delta g_{7} / g_{7} \simeq 0.0001739775786$. As a consequence, correcting these terms does not affect any of the significant conclusions we reached in [3] which were mainly aimed at describing strong field effects. In particular, our fits Eqs. (5.11) and (6.39) did not make any use of the $y^{7}$ and $y^{8}$ coefficients but only relied on $3 \mathrm{PN}$ information and on the strong field numerical data of [2].

Finally, we have introduced here a new method for analytically computing the subtraction terms of self-force quantities. Instead of the traditionally used Hadamardlike near-world-line singularity expansions, our new method is based on a WKB analysis of both the radial and angular equations satisfied by the metric, when considering them in the limit $l \rightarrow \infty$ with the ratio $m / l$ fixed. We have shown on two examples $\left(h_{k k}\right.$ and the spin pre- 
cession) that our method leads rather simply to closed form expressions for the subtraction terms.
Appendix A: Higher PN terms in $g_{S_{*}}^{1 S F}$

Combining the $O\left(y^{10.5}\right)$-accurate computation of $\delta(y)$ above with our recent $O\left(y^{10.5}\right)$-accurate computation of the main effective one-body radial potential $a(u)$ [15], we can raise the $\mathrm{PN}$ expansion order of the effective gyromagnetic ratio $g_{S_{*}}^{1 \mathrm{SF}}$ from the $O\left(u^{7.5}\right)$ level given in Eq. (6.37) of 3] to the $O\left(u^{9.5}\right)$ level. We list below the final result, expressed in the effective one-body radial variable $u$. 


$$
\begin{aligned}
& g_{S_{*}}^{1 \mathrm{SF}}(u)=-\frac{3}{4} u-\frac{39}{4} u^{2}+\left(\frac{41}{32} \pi^{2}-\frac{7627}{192}\right) u^{3} \\
& +\left(-48 \gamma+\frac{23663}{2048} \pi^{2}-\frac{1456}{15} \ln (2)-\frac{1017}{20}-24 \ln (u)\right) u^{4} \\
& +\left(-\frac{729}{7} \ln (3)+\frac{9832}{35} \gamma+\frac{712905}{4096} \pi^{2}+\frac{70696}{105} \ln (2)-\frac{161160813}{89600}+\frac{4916}{35} \ln (u)\right) u^{5} \\
& -\frac{93304}{1575} \pi u^{11 / 2} \\
& +\left(\frac{315657}{280} \ln (3)+\frac{480829}{2835} \gamma+\frac{16790137}{1048576} \pi^{4}-\frac{674904611}{7077888} \pi^{2}-\frac{2954531}{2835} \ln (2)-\frac{18167439833}{7257600}+\frac{480829}{5670} \ln (u)\right) u^{6} \\
& +\frac{4596019}{12600} \pi u^{13 / 2} \\
& +\left(-\frac{12227517}{3080} \ln (3)-1088 \zeta(3)-\frac{1953125}{3564} \ln (5)-\frac{903605468}{121275} \gamma+\frac{58208}{105} \gamma^{2}-\frac{204902966117}{335544320} \pi^{4}\right. \\
& +\frac{1167584}{525} \ln (2)^{2}-\frac{7532631301}{9175040} \pi^{2}+\frac{499904}{225} \ln (2) \gamma-\frac{5587843424}{779625} \ln (2)+\frac{48146264595158227}{625895424000} \\
& \left.-\frac{451802734}{121275} \ln (u)+\frac{58208}{105} \gamma \ln (u)+\frac{249952}{225} \ln (2) \ln (u)+\frac{14552}{105} \ln (u)^{2}\right) u^{7} \\
& +\frac{118299749}{2182950} \pi u^{15 / 2} \\
& +\left(-\frac{52964727700527}{3139136000} \ln (3)+\frac{141648}{35} \zeta(3)+\frac{366384765625}{41513472} \ln (5)+\frac{4204284206047}{264864600} \gamma-\frac{10974904}{3675} \gamma^{2}\right. \\
& -\frac{1135089788764019}{42949672960} \pi^{4}-\frac{20022888}{1225} \ln (2)^{2}+\frac{142155}{49} \gamma \ln (3)+\frac{142155}{49} \ln (2) \ln (3)+\frac{1241427590810221}{369937612800} \pi^{2} \\
& -\frac{164036944}{11025} \ln (2) \gamma+\frac{90305230479881}{3972969000} \ln (2)+\frac{142155}{98} \ln (3)^{2}+\frac{1094977266529990589159}{427173626880000} \\
& \left.+\frac{4189028005087}{529729200} \ln (u)-\frac{10974904}{3675} \gamma \ln (u)-\frac{82018472}{11025} \ln (2) \ln (u)+\frac{142155}{98} \ln (u) \ln (3)-\frac{2743726}{3675} \ln (u)^{2}\right) u^{8} \\
& +\left(\frac{142517152}{55125} \ln (2) \pi-\frac{6965217870900563}{762810048000} \pi+\frac{213592544}{165375} \pi \gamma-\frac{1996192}{4725} \pi^{3}+\frac{106796272}{165375} \pi \ln (u)\right) u^{17 / 2} \\
& +\left(\frac{15312301495292259}{69060992000} \ln (3)+\frac{1294640}{81} \zeta(3)-\frac{13759767578125}{249080832} \ln (5)+\frac{16569352454284793}{202280878800} \gamma\right. \\
& -\frac{4477353976}{1403325} \gamma^{2}-\frac{691974898583334793}{5497558138880} \pi^{4}+\frac{540743945464}{9823275} \ln (2)^{2}-\frac{847418031}{26950} \gamma \ln (3)-\frac{847418031}{26950} \ln (2) \ln (3) \\
& -\frac{1368675122796890401}{146495294668800} \pi^{2}+\frac{264895105264}{9823275} \ln (2) \gamma-\frac{207604582525}{402653184} \pi^{6}-\frac{3003559322617}{1111968000} \ln (7) \\
& -\frac{78318056677502249}{7079830758000} \ln (2)-\frac{847418031}{53900} \ln (3)^{2}+\frac{849725095980588589949507303}{66987659472814080000}+\frac{16550580788732153}{404561757600} \ln (u) \\
& \left.-\frac{4477353976}{1403325} \gamma \ln (u)+\frac{132447552632}{9823275} \ln (2) \ln (u)-\frac{847418031}{53900} \ln (u) \ln (3)-\frac{1119338494}{1403325} \ln (u)^{2}\right) u^{9} \\
& +\left(-\frac{78150479}{4410} \ln (2) \pi+\frac{298035034972802327}{11783531760000} \pi-\frac{1137638861}{154350} \pi \gamma+\frac{4065633}{1372} \pi \ln (3)+\frac{23679911}{13230} \pi^{3}\right. \\
& \left.-\frac{1137638861}{308700} \pi \ln (u)\right) u^{19 / 2}+O_{\ln }\left(u^{10}\right) \text {. }
\end{aligned}
$$

Here the coefficients of $u^{6}$ and $u^{7}$ differ from the ones given in Ref. [3] because of the corresponding change in $\delta \psi$. More precisely, the terms to be added to Eqs. $(6.36)_{1}$ and $(6.37)_{1}$ in [3] read

$$
\Delta c_{6}^{g_{S *}}=\Delta c_{7}^{\delta \psi}
$$

$$
\Delta c_{7}^{g_{S *}}=\Delta c_{8}^{\delta \psi}-\frac{3}{2} \Delta c_{7}^{\delta \psi}
$$


Let us also point out a misprint in the expression of $g_{7}^{\ln }$ given in Eq. $(6.37)_{2}$ of [3]: the additional term

$$
+\frac{249952}{225} \ln 2
$$

was inadvertently omitted.

Acknowledgments
We thank Sam Dolan, Patrick Nolan, Adrian Ottewill, Niels Warburton, Barry Wardell and Chris Kavanagh for useful discussions, exchange of data and constructive criticism. We also thank Abhay Shah for informative email exchanges. Finally we are grateful to ICRANet for partial support. D.B. thanks the Italian INFN (Naples) for partial support and IHES for hospitality during the development of this project.
[1] T. Damour, P. Jaranowski and G. Schaefer, "Hamiltonian of two spinning compact bodies with next-to-leading order gravitational spin-orbit coupling," Phys. Rev. D 77, 064032 (2008) arXiv:0711.1048 [gr-qc]].

[2] S. R. Dolan, N. Warburton, A. I. Harte, A. Le Tiec, B. Wardell and L. Barack, "Gravitational self-torque and spin precession in compact binaries," Phys. Rev. D 89, no. 6, 064011 (2014) arXiv:1312.0775 [gr-qc]].

[3] D. Bini and T. Damour, "Two-body gravitational spinorbit interaction at linear order in the mass ratio," Phys. Rev. D 90, no. 2, 024039 (2014) arXiv:1404.2747 [gr-qc]].

[4] S. L. Detweiler, "A Consequence of the gravitational selfforce for circular orbits of the Schwarzschild geometry," Phys. Rev. D 77, 124026 (2008) arXiv:0804.3529 [gr-qc]].

[5] S. R. Dolan, P. Nolan, A. Ottewill, N. Warburton, B. Wardell, C. Kavanagh, private communication.

[6] S. R. Dolan, P. Nolan, A. C. Ottewill, N. Warburton and B. Wardell, "Tidal invariants for compact binaries on quasicircular orbits," Phys. Rev. D 91, no. 2, 023009 (2015) arXiv:1406.4890 [gr-qc]].

[7] A. Shah, private communication.

[8] A. G. Shah, J. L. Friedman and B. F. Whiting, "Finding high-order analytic post-Newtonian parameters from a high-precision numerical self-force calculation," Phys. Rev. D 89, no. 6, 064042 (2014) arXiv:1312.1952 [gr-qc]].

[9] S. L. Detweiler and B. F. Whiting, "Selfforce via a Green's function decomposition," Phys. Rev. D 67, 024025 (2003) gr-qc/0202086.

[10] N. Straumann, General Relativity (Second Edition), Ed. Springer (Dordrecht, 2013)

[11] D. Bini and T. Damour, "Analytical determination of the two-body gravitational interaction potential at the fourth
post-Newtonian approximation," Phys. Rev. D 87, no. 12, 121501 (2013) arXiv:1305.4884 [gr-qc]].

[12] D. Bini and T. Damour, "High-order post-Newtonian contributions to the two-body gravitational interaction potential from analytical gravitational self-force calculations," Phys. Rev. D 89, no. 6, 064063 (2014) arXiv:1312.2503 [gr-qc]].

[13] D. Bini and T. Damour, "Analytic determination of the eight-and-a-half post-Newtonian self-force contributions to the two-body gravitational interaction potential," Phys. Rev. D 89, no. 10, 104047 (2014) arXiv:1403.2366 [gr-qc]].

[14] D. Bini and T. Damour, "Gravitational self-force corrections to two-body tidal interactions and the effective one-body formalism," Phys. Rev. D 90, no. 12, 124037 (2014) arXiv:1409.6933 [gr-qc]].

[15] D. Bini and T. Damour, "Detweiler's gauge-invariant redshift variable: analytic determination of the nine and nine-and-a-half post-Newtonian self-force contributions," arXiv:1502.02450 [gr-qc].

[16] L. Barack, Y. Mino, H. Nakano, A. Ori and M. Sasaki, "Calculating the gravitational selfforce in Schwarzschild space-time," Phys. Rev. Lett. 88, 091101 (2002) gr-qc/0111001.

[17] S. L. Detweiler, E. Messaritaki and B. F. Whiting, "Selfforce of a scalar field for circular orbits about a Schwarzschild black hole," Phys. Rev. D 67, 104016 (2003) gr-qc/0205079

[18] A. G. Shah, T. S. Keidl, J. L. Friedman, D. H. Kim and L. R. Price, "Conservative, gravitational self-force for a particle in circular orbit around a Schwarzschild black hole in a Radiation Gauge," Phys. Rev. D 83, 064018 (2011) arXiv:1009.4876 [gr-qc]]. 